PROCEEDINGS OF THE

AMERICAN MATHEMATICAL SOCIETY

Volume 133, Number 2, Pages 627-631

S 0002-9939(04)07667-1

Article electronically published on September 8, 2004

\title{
REGULARITY OF LOOP GROUP FACTORIZATION
}

\author{
MICHAEL TAYLOR
}

(Communicated by Andreas Seeger)

\begin{abstract}
In the factorization of a $\operatorname{Gl}(n, \mathbb{C})$-valued loop $\varphi$ into a unitary factor and a factor holomorphic in the disk, it is shown that the two factors each have as much regularity as $\varphi$, measured in a variety of function spaces, though with exceptions. This is analogous to known results for Birkhoff factorization, but somewhat different techniques are involved.
\end{abstract}

\section{InTRODUCTION}

A well-known and useful result in the theory of loop groups is the fact that any (sufficiently regular) function $\varphi: \mathbb{T}^{1} \rightarrow \operatorname{Gl}(n, \mathbb{C})$ can be factored as

$$
\varphi(\theta)=u(\theta) h\left(e^{i \theta}\right),
$$

where

$$
u: \mathbb{T}^{1} \rightarrow \mathrm{U}(n), \quad h: \bar{\Delta} \rightarrow \mathrm{Gl}(n, \mathbb{C}), \text { holomorphic on } \Delta .
$$

Here $\mathbb{T}^{1}=\mathbb{R} /(2 \pi \mathbb{Z})$ and $\bar{\Delta}=\{z \in \mathbb{C}:|z| \leq 1\}$, with interior $\Delta$. The factorization (1.1) is unique if one requires $u(0)=I$.

It is also well known that $u$ is $C^{\infty}$ provided $\varphi$ is $C^{\infty}$. Here we investigate how smooth $u$ is if $\varphi$ has some limited regularity. The basic result will be that $u$ is as smooth as $\varphi$, when measured in a variety of function spaces, though with some exceptions.

As a guide to what results to hope for, we first look at the case $n=1$, where the factorization (1.1) is straightforward. First, if $\ell$ is the winding number of $\varphi: \mathbb{T}^{1} \rightarrow \mathbb{C} \backslash 0$, we can write $\varphi(\theta)=e^{i \ell \theta} e^{\psi(\theta)}$, with a continuous $\psi$, uniquely determined up to an additive constant, an integer multiple of $2 \pi i$. Then, using

$$
P_{+} \sum_{\nu \in \mathbb{Z}} c_{\nu} e^{i \nu \theta}=\sum_{\nu \geq 0} c_{\nu} e^{i \nu \theta}, \quad P_{-}=I-P_{+},
$$

we can write

$$
\psi(\theta)=\psi_{0}(\theta)+\psi_{1}(\theta)=\left(P_{-} \psi-\overline{P_{-} \psi}\right)+\left(P_{+} \psi+\overline{P_{-} \psi}\right),
$$

and the factorization (1) is achieved with

$$
u(\theta)=e^{i \ell \theta+\psi_{0}(\theta)}, \quad h\left(e^{i \theta}\right)=e^{\psi_{1}(\theta)} .
$$

Received by the editors October 23, 2003.

2000 Mathematics Subject Classification. Primary 22E67, 35S05.

This work was partially supported by the National Science Foundation.

(C)2004 American Mathematical Society Reverts to public domain 28 years from publication 
Well-known mapping properties of $P_{+}$guarantee that

$$
\varphi \in C^{r}\left(\mathbb{T}^{1}\right) \Longrightarrow u \in C^{r}\left(\mathbb{T}^{1}\right), \quad r \in \mathbb{R}^{+} \backslash \mathbb{Z}^{+} .
$$

On the other hand, if $k$ is a positive integer, we have the weaker result

$$
\varphi \in C^{k}\left(\mathbb{T}^{1}\right) \Longrightarrow u^{(k)} \in \operatorname{bmo}\left(\mathbb{T}^{1}\right) .
$$

We also have

$$
\varphi \in C_{*}^{k}\left(\mathbb{T}^{1}\right) \Longrightarrow u \in C_{*}^{k}\left(\mathbb{T}^{1}\right),
$$

where $C_{*}^{r}$ denotes the scale of Zygmund spaces. Our goals here include establishing such implications when $n>1$.

In $\S 2$ we recall the construction of the factorization (1.1), in a form that is a convenient variant to the presentation of [2], and we reduce the study of the regularity of the factor $u$ to the regularity of a certain linear function space $V_{0}$. In $\S 3$ we analyze the regularity of elements of $V_{0}$ in terms of mapping properties of commutators of certain singular integral operators and multiplication operators. Our conclusions on the regularity of the factors in (1.1) are presented in Proposition 3.3 .

\section{Construction of the factorization}

We give a construction of the factorization (1.1), adapted from [2], making use of the action of $\varphi$ on the Hilbert space $L^{2}\left(\mathbb{T}^{1}, \mathbb{C}^{n}\right)$. Let us write

$$
U, M: L^{2}\left(\mathbb{T}^{1}, \mathbb{C}^{n}\right) \rightarrow L^{2}\left(\mathbb{T}^{1}, \mathbb{C}^{n}\right), \quad U f(\theta)=e^{i \theta} f(\theta), M f(\theta)=\varphi(\theta) f(\theta),
$$

assuming $\varphi: \mathbb{T}^{1} \rightarrow \operatorname{Gl}(n, \mathbb{C})$ is continuous, or more generally $\varphi$ and $\varphi^{-1}$ are bounded and measurable. Next, let us set

$$
H^{(j)}=\left\{\sum_{k \geq j} c_{k} e^{i k \theta}: c_{k} \in \mathbb{C}^{n}, \sum\left|c_{k}\right|^{2}<\infty\right\},
$$

so we have a filtration $\cdots \supset H^{(j)} \supset H^{(j+1)} \supset \cdots$, and $U^{\nu}: H^{(j)} \rightarrow H^{(j+\nu)}, j, \nu \in$ $\mathbb{Z}$. Now set

$$
W^{(j)}=M H^{(j)},
$$

so we have a filtration $\cdots \supset W^{(j)} \supset W^{(j+1)} \supset \cdots$, and

$$
U^{\nu}: W^{(j)} \longrightarrow W^{(j+\nu)}, \quad j, \nu \in \mathbb{Z}
$$

Set

$$
V_{j}=W^{(j)} \ominus W^{(j+1)} ;
$$

each $V_{j}$ is an $n$-dimensional subspace of $L^{2}\left(\mathbb{T}^{1}, \mathbb{C}^{n}\right)$, and

$$
U^{\nu}: V_{j} \longrightarrow V_{j+\nu}, \quad j, \nu \in \mathbb{Z}
$$

by (2.4) plus unitarity. Furthermore, the spaces $V_{j}$ are mutually orthogonal, and their linear span is dense in $L^{2}\left(\mathbb{T}^{1}, \mathbb{C}^{n}\right)$.

Let

$$
v_{01}, \ldots, v_{0 n} \in V_{0}
$$

be an orthonormal basis of $V_{0}$. Then

$$
v_{j 1}=U^{j} v_{01}, \ldots, v_{j n}=U^{j} u_{0 n} \in V_{j}
$$


is an orthonormal basis of $V_{j}$ for each $j \in \mathbb{Z}$, so $\left\{v_{j k}: j \in \mathbb{Z}, 1 \leq k \leq n\right\}$ is an orthonormal basis of $L^{2}\left(\mathbb{T}^{1}, \mathbb{C}^{n}\right)$. Note that $v_{j k}(\theta)$ is a function of $\theta$ with values in $\mathbb{C}^{n}$, which we interpret as a space of column vectors. Form the $n \times n$ matrix function

$$
u_{0}(\theta)=\left(v_{01}(\theta), \ldots, v_{0 n}(\theta)\right) .
$$

Note that

$$
U^{j} u_{0}(\theta)=u_{j}(\theta)=\left(v_{j 1}(\theta), \ldots, v_{j n}(\theta)\right) .
$$

Thus if we consider

$$
U_{0}: L^{2}\left(\mathbb{T}^{1}, \mathbb{C}^{n}\right) \rightarrow L^{2}\left(\mathbb{T}^{1}, \mathbb{C}^{n}\right), \quad U_{0} f(\theta)=u_{0}(\theta) f(\theta),
$$

we see that $U_{0}$ maps the orthonormal basis $\left\{e_{k} e^{i j \theta}: j \in \mathbb{Z}, 1 \leq k \leq n\right\}$ of $L^{2}\left(\mathbb{T}^{1}, \mathbb{C}^{n}\right)$ $\left(e_{1}, \ldots, e_{n}\right.$ denoting the standard basis of $\left.\mathbb{C}^{n}\right)$ to the orthonormal basis $\left\{v_{j k}: j \in\right.$ $\mathbb{Z}, 1 \leq k \leq n\} ;$ so $U_{0}$ is unitary. Hence

$$
u_{0}(\theta) \in \mathrm{U}(n),
$$

for almost all $\theta \in \mathbb{T}^{1}$. While $(2.9)$ a priori yielded $u_{0} \in L^{2}\left(\mathbb{T}^{1}, M(n, \mathbb{C})\right)$, we now have $u_{0} \in L^{\infty}\left(\mathbb{T}^{1}, M(n, \mathbb{C})\right)$. Furthermore, $u(\theta)=u_{0}(\theta)$ is the factor that appears in (1.1), as a consequence of the fact that $U_{0}^{-1} M$ and $M^{-1} U_{0}$ preserve the subspace $H^{(0)} \subset L^{2}\left(\mathbb{T}^{1}, \mathbb{C}^{n}\right)$.

\section{Regularity}

From here, establishing regularity of the unitary factor $u(\theta)$ in (1.1) given regularity of $\varphi(\theta)$ comes down to establishing regularity of elements of the space $V_{0}=W^{(0)} \ominus W^{(1)} \subset L^{2}\left(\mathbb{T}^{1}, \mathbb{C}^{n}\right)$. We have already seen that

$$
V_{0} \subset L^{\infty}\left(\mathbb{T}^{1}, \mathbb{C}^{n}\right),
$$

whenever $\varphi$ is continuous.

To analyze $V_{0}$ further, we make use of $P_{+}^{(j)}$, the orthogonal projection of $L^{2}\left(\mathbb{T}^{1}, \mathbb{C}^{n}\right)$ onto $H^{(j)}$, i.e.,

$$
P_{+}^{(j)} \sum_{\nu \in \mathbb{Z}} c_{\nu} e^{i \nu \theta}=\sum_{\nu \geq j} c_{\nu} e^{i \nu \theta}
$$

and $P_{-}^{(j)}=I-P_{+}^{(j)}$. Note that $v \in W^{(0)}$ if and only if $v=M f$ for some $f \in H^{(0)}$, so

$$
v \in W^{(0)} \Longrightarrow P_{-}^{(0)} v=\left[P_{-}^{(0)}, M\right] f
$$

where $[A, B]=A B-B A$ is the commutator. Note that $f(\theta)=\varphi(\theta)^{-1} v(\theta) \in$ $L^{\infty}\left(\mathbb{T}^{1}, \mathbb{C}^{n}\right)$, if $v \in V_{0}$, by (3.1). Next, since $W^{(1)}$ is the range of $M P_{+}^{(1)}$,

$$
\begin{aligned}
v \perp W^{(1)} & \Longrightarrow P_{+}^{(1)} M^{*} v=0 \\
& \Longrightarrow P_{+}^{(1)} v=\left(M^{*}\right)^{-1}\left[M^{*}, P_{+}^{(1)}\right] v .
\end{aligned}
$$

Now $v=P_{-}^{(0)} v+\Pi_{0} v+P_{+}^{(1)} v$, where $\Pi_{0}$ denotes the orthogonal projection of $L^{2}\left(\mathbb{T}^{1}, \mathbb{C}^{n}\right)$ onto the space of constant functions. Putting together these observations yields the following lemma. 
Lemma 3.1. If $v \in V_{0}$, then $v \in L^{\infty}\left(\mathbb{T}^{1}, \mathbb{C}^{n}\right)$ and

$$
v=\left[P_{-}^{(0)}, M\right] M^{-1} v+\Pi_{0} v+\left(M^{*}\right)^{-1}\left[M^{*}, P_{+}^{(1)}\right] v .
$$

Recall that $M$ is given in (2.1), and hence

$$
M^{*} f(\theta)=\varphi(\theta)^{*} f(\theta), \quad M^{-1} f(\theta)=\varphi(\theta)^{-1} f(\theta) .
$$

Now regularity results on elements of $V_{0}$ follow from mapping properties of the commutators that appear in (3.5). In our statement of mapping properties, we use the function spaces appearing in (1.6)-(1.8), and also the $L^{p}$-Sobolev spaces $H^{s, p}\left(\mathbb{T}^{1}\right)$, with $p \in(1, \infty)$, and the bmo-Sobolev spaces $\mathfrak{h}^{r, \infty}\left(\mathbb{T}^{1}\right)$, consisting of functions with $r$ derivatives in bmo. Some of the following results may be well known and some not; all are proven in $\S 5$ of [3].

Lemma 3.2. Let $M f(\theta)=\varphi(\theta) f(\theta)$, and let $P$ have the form $P_{+}^{(j)}$ or $P_{-}^{(j)}$ for some $j$. Then the following commutator estimates hold:

$$
\begin{aligned}
\|[M, P] v\|_{C_{*}^{r}} & \leq C\|\varphi\|_{C_{*}^{r}}\left(\|v\|_{L^{\infty}}+\|P v\|_{L^{\infty}}\right), \quad r>0, \\
\|[M, P] v\|_{H^{r, p}} & \leq C\|\varphi\|_{\mathfrak{h}^{r, \infty}}\|v\|_{L^{p}}, \quad r>0,1<p<\infty, \\
\|[M, P] v\|_{\mathfrak{h}^{r, \infty}} & \leq C\|\varphi\|_{\mathfrak{h}^{r, \infty}}\left(\|v\|_{L^{\infty}}+\|P v\|_{L^{\infty}}\right), \quad r>0, \\
\|[M, P] v\|_{H^{r, p}} & \leq C\|\varphi\|_{H^{r, p}}\left(\|v\|_{L^{\infty}}+\|P v\|_{L^{\infty}}\right), \quad r>0,1<p<\infty .
\end{aligned}
$$

These results lead to the following conclusion about the factorization (1.1).

Proposition 3.3. Given $\varphi: \mathbb{T}^{1} \rightarrow \operatorname{Gl}(n, \mathbb{C}), n \geq 1$, the factorization (1.1)-(1.2) has the following behavior:

$$
\begin{gathered}
\varphi \in C_{*}^{r}, r>0 \Longrightarrow u \in C_{*}^{r}, \\
\varphi \in \mathfrak{h}^{r, \infty}, r>0 \Longrightarrow u \in \mathfrak{h}^{r, \infty} .
\end{gathered}
$$

Furthermore, assuming $\varphi$ and $\varphi^{-1}$ are bounded and measurable, and $1<p<\infty$,

$$
\varphi \in H^{r, p}, r>0 \Longrightarrow u \in H^{r, p} .
$$

Proof. We need to show that all $v \in V_{0}$ have the asserted regularity. We already know $v \in L^{\infty}\left(\mathbb{T}^{1}, \mathbb{C}^{n}\right)$. Thus (3.8) applies to give $v \in H^{s, p}$ for all $p<\infty$, all $s<r$, under either of the hypotheses in (3.11)-(3.12). This is more than enough to give $P_{+}^{(1)} v \in L^{\infty}\left(\mathbb{T}^{1}, \mathbb{C}^{n}\right)$ and $P_{-}^{(0)}\left(M^{-1} v\right) \in L^{\infty}\left(\mathbb{T}^{1}, \mathbb{C}^{n}\right)$, so $(3.7)$ and (3.9) are applicable to the right side of (3.5). We have $\left[P_{-}^{(0)}, M\right] M^{-1} v$ and $\left[M^{*}, P_{+}^{(1)}\right] v$ in $C_{*}^{r}$ (resp., $\left.\mathfrak{h}^{r, \infty}\right)$, given $\varphi \in C_{*}^{r}$ (resp., $\left.\mathfrak{h}^{r, \infty}\right), r>0$. Finally, the spaces $C_{*}^{r}$ and $\mathfrak{h}^{r, \infty}$ are all algebras, for $r>0$, with the property

$$
v \in \mathcal{A}, \quad \frac{1}{v} \in L^{\infty} \Longrightarrow \frac{1}{v} \in \mathcal{A} .
$$

So $\left(M^{*}\right)^{-1}\left[M^{*}, P_{+}^{(1)}\right] v$ also belongs to the desired space, in the setting of (3.11) or (3.12).

A similar argument, bringing in (3.10), also establishes (3.13). In this case one uses the fact that $L^{\infty}\left(S^{1}\right) \cap H^{r, p}\left(S^{1}\right)$ is an algebra, with property (3.14).

Remark 3.1. Clearly $h\left(e^{i \theta}\right)$ inherits the same degree of regularity as $u(\theta)$ in the cases covered by Proposition 3.3. We mention that the special case of (3.13) in which $p=2$ and $r=1 / 2$ was treated in [2]. 
Remark 3.2. There are similar regularity results for the Birkhoff factorization. In this case the familiar Riemann-Hilbert approach applies, and so such results are well known; cf. [1].

\section{REFERENCES}

1. K. Clancey and I. Gohberg, Factorization of Matrix Functions and Singular Integral Operators, Birkhäuser, Boston, 1981. MR 0657762 (84a:47016)

2. A. Pressley and G. Segal, Loop Groups, Oxford Univ. Press, 1986. MR 0900587 (88i:22049)

3. M. Taylor, Commutator estimates for Hölder continuous multipliers and variants, Preprint, 2003.

Department of Mathematics, University of North Carolina, Chapel Hill, North CarOLINA 27599

E-mail address: met@math.unc.edu 